\title{
Anesthetic management of an adult patient with Rett syndrome and limited mouth opening
}

\section{-A case report-}

\author{
Ji-Sung Nho, Dong-Shik Shin, Jee-Youn Moon, Jae-Woo Yi, Jong-Man Kang, Bong-Jae Lee, \\ Dong-Ok Kim, and Jun-Young Chung
}

Department of Anesthesiology and Pain Medicine, Kyung Hee University Hospital at Gangdong, College of Medicine, Kyung Hee University, Seoul, Korea

Rett syndrome is a neurological disease that occurs only in females and it manifests with mental retardation, seizures, movement disorders, autistic behavior and abnormal breathing. A 19-year-old female with Rett syndrome underwent ophthalmologic surgery under general anesthesia at our institution. Airway control was difficult due to her limited mouth opening. We recommend that anesthesiologists should have proper knowledge about this disease and the patients to avoid the complications and problems that can be encountered during the perioperative period. (Korean J Anesthesiol 2011; 61: 428-430)

Key Words: Bronchoscopic intubation, Limited mouth opening, Rett syndrome.

Rett syndrome is a progressive neurological disorder that affects only girls and it presents many challenges to anesthesiologists. The syndrome is inherited and the gene is carried on the X chromosome. Rett syndrome is characterized by mental retardation, convulsions, autistic behavior and scoliosis, and all this can cause abnormal breathing [1]. Bruxism, oculogyric crises, parkinsonism and dystonia are also common, while myoclonus and choreoathetosis are seen infrequently [2]. These features lead to the need for special care and preparation by anesthesiologists.

We recently anesthetized a patient who had limited mouth opening and she demonstrated a few other features of Rett syndrome. The anesthetic experience with this type of case has not been previously reported in Korea.

\section{Case Report}

A 19-year-old girl with Rett syndrome was scheduled for elective phacoemulsification with intraocular lens insertion for immature cataracts. We did not know about her family history and other medical history because she was an orphan and she could not communicate with our medical team. But there was some description on the consultation paper from her orphanage. According to the paper, her physical disability had

Received: October 15, 2010. Revised: 1st, December 31, 2010; 2nd, March 31, 2011. Accepted: April 4, 2011.

Corresponding author: Jun-Young Chung, M.D., Department of Anesthesiology and Pain Medicine, Kyung Hee University Hospital at Gangdong, College of Medicine, Kyung Hee University, 149, Sangil-dong, Kangdong-gu, Seoul 134-090, Korea. Tel: 82-2-440-6191, Fax: 82-2-440-7808, E-mail: madsleep@naver.com

(c) This is an open-access article distributed under the terms of the Creative Commons Attribution Non-Commercial License (http:// creativecommons.org/licenses/by-nc/3.0/), which permits unrestricted non-commercial use, distribution, and reproduction in any medium, provided the original work is properly cited. 
steadily progressed although her developmental milestones had been normal until the age of ten months, and she was known to have diffuse brain atrophy with mental retardation, including typical autistic disorder and convulsions. Other features of her disorder were bruxism and trismus. She also had untreated scoliosis and she had been bedridden for 5 years.

On examination, her weight was $23 \mathrm{~kg}$ (below the 5 th percentile), her body temperature and blood pressure were normal and she was not distressed. The thyromental distance was $3.5 \mathrm{~cm}$ and the airway was normal except for trismus and excessive secretions. Auscultation of the heart and lungs was unremarkable. She exhibited hand-wringing and her muscle mass was diminished. Her muscle tone was increased and her reflexes were brisk. Her seizures were well controlled with valproate.

The preoperative hemoglobin level was $11.0(\mathrm{~g} / \mathrm{dl})$ and the other lab tests were normal. On chest x-ray, the heart and lungs were unremarkable, although severe scoliosis of the T/L-spine and diffuse osteoporosis were observed. Pulmonary function testing was not conducted due to a lack of patient cooperation. A head CT scan revealed diffuse brain atrophy, and the background was slow on the EEG.

The patient was quiet on arrival to the operating room without premedication. In the operating room, 3-lead ECG, noninvasive blood pressure monitoring and a pulse oximetry monitor were placed. Atropine $0.2 \mathrm{mg}$ was given as premedication. She was continuously grinding her teeth and could not communicate. Her pulse oximetry was $99 \%$ on room air and her blood pressure was 110/75 mmHg. Anesthesia was then intravenously induced with propofol $40 \mathrm{mg}$ and rocuronium 15 mg. After loss of consciousness, mask ventilation was started with 2 vol\% of sevoflurane. Because we expected her trismus would be improved after injection of rocuronium, direct laryngoscopy was attempted. But while the patient was fully

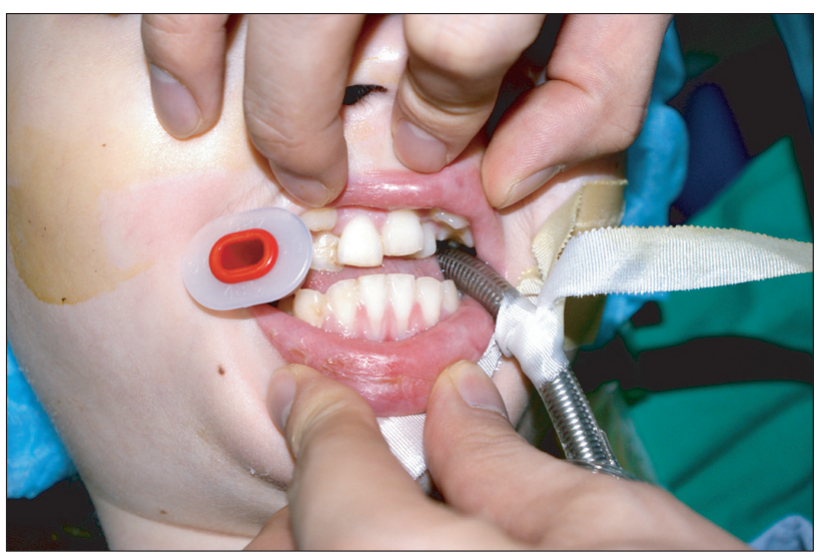

Fig. 1. This show limited mouth opening and oral endotracheal tube was intubated via the small space of malocclusion. relaxed, her mouth opening was very limited. We could not introduce a Macintosh laryngoscope (No. 2) into the mouth. So, the fiberoptic-guided nasotracheal technique was initially chosen considering the extremely limited mouth opening, but this was unsuccessful because her nostrils were too small to intubate with a tube with an internal diameter of $5.0 \mathrm{~mm}$. Before an attempt was made with a $4.5 \mathrm{~mm}$ tube, we realized that the small space due to the malocclusion was large enough to handle a 480 -sized oral airway (diameter: $0.48 \mathrm{~mm}$ ). Fiberopticguided orotracheal intubation through this space was then successfully performed with suction (Fig. 1). The trachea was intubated with a $5.0 \mathrm{~mm}$ oral tracheal tube and it was fixed at 18 $\mathrm{cm}$. Anesthesia was maintained with $\mathrm{N}_{2} \mathrm{O} 2 \mathrm{~L} / \mathrm{min}, \mathrm{O}_{2} 2 \mathrm{~L} / \mathrm{min}$, sevoflurane $1.0-2.0 \mathrm{vol} \%$, a tidal volume of $200 \mathrm{ml}$ and a $\mathrm{RR}$ of $18 / \mathrm{min}$.

After the completion of surgery, which lasted 1 hour and there were no complications, the nitrous oxide and sevoflurane were discontinued, and the muscle relaxant was reversed with glycopyrrolate $0.2 \mathrm{mg}$ and pyridostigmine $10 \mathrm{mg}$. We tried to wean her from the ventilator, but her recovery was slow and complicated by episodes of shaking. After 20 minutes, the patient began spontaneous breathing. The patient was then transferred to the PACU after her respiration was found to be adequate. She remained drowsy in the PACU and it took a full day for her to regain full consciousness.

\section{Discussion}

Rett syndrome is characterized by a devastating deterioration of the central nervous system in young females and it is accompanied by neurological problems that are associated with failure to thrive. There is a lack of sufficient data to make reliable estimates about the life expectancy beyond the age of 40 , although the average life expectancy of a girl with Rett syndrome may be in the mid-40s [3].

Patients with Rett syndrome have normal a growth and development pattern after birth. But many clinical features arise during the age of 6 to 18 months. Microcephaly due to growth retardation, diminished muscle tone, decreased eye contact and inattentive behavior develop during this period. As the patients grow up, their mental retardation and autistic features are worsen. Immobility, respiratory dysrhythmia (hyperventilation, periodic apnea), gastrointestinal dysfunctions (peristalsis, esophageal atony, obstipation, etc.), spasticity, joint contraction and arrhythmias due to cardiac transduction defects also appear with disease progression [4].

So, there are several points to consider for the anesthetic management of Rett syndrome patients. The presence of scoliosis and muscular tonicity and the breathing abnormalities that range from centrally mediated hyperventilation to apnea 
[5] make it necessary to evaluate the respiratory status using oxygen saturation to predict the outcomes before surgery [6]. There is also a great risk of aspiration pneumonia during anesthetic induction or emergence due to esophageal atony.

There are no reports about anesthetic experiences in patients with Rett syndrome and who have limited mouth opening. But joint contraction and spasticity also can cause many problems. It can cause difficulties to correctly position the patient and if it involves the TM joint like our case, the anesthesiologist can be confronted with problems during orotracheal intubation or airway management. There are many reports on airway management in patients with limited mouth opening. Antegrade fiberoptic-guided orotracheal or nasotracheal intubation, retrograde guidewire-assisted fiberoptic intubation and blind nasal intubation can be considered $[7,8]$. Fortunately for us, antegrade fiberoptic-guided orotracheal intubation was possible to perform by an experienced anesthesiologist. Yet we also prepared a tracheostomy set to secure the airway in the event of severe difficulty when securing the airway.

Preoperatively evaluating a Rett syndrome patient is very important. We should check the breathing patterns, the preoperative arterial blood gases, the presence of gastrointestinal reflux and autonomic disturbances, the seizure history, the ECG and the musculoskeletal system. We should carefully interview the patient and the guardians for obtain proper information about the patient. However, the mental retardation and autistic behavior of these patients make this process difficult.

These patients tend to highly sensitive to analgesia, sedatives, and anesthetic agents $[9,10]$. Konarzewski \& Misso reported delayed awakening from anesthesia in Rett syndrome patients [11]. They thought that patients with Rett syndrome may be unduly sensitive to sedative drugs and they exhibit slow recovery from anesthesia. Our case also exhibited delayed recovery. So, the anesthesiologist should have great concern about the choice of anesthetics and maintaining the proper depth of anesthesia.
In conclusion, we experienced great difficulty securing the airway in an adult female patient with Rett syndrome during minor surgery, and this is the first report about the anesthetic management of a patient with Rett syndrome. The patient with Rett syndrome has many difficulties and points to be considered for anesthetic management, as described above. Anesthesiologists should have proper knowledge about the disease and the patients to avoid the complications and problems that can be encountered during the perioperative and postoperative periods.

\section{References}

1. Hagberg B. Rett syndrome: clinical peculiarities and biological mysteries. Acta Paediatr 1995; 84: 971-6.

2. FitzGerald PM, Jankovic J, Percy AK. Rett syndrome and associated movement disorders. Mov Disord 1990; 5: 195-202.

3. Chahrour M, Zoghbi HY. The story of Rett syndrome: from clinic to neurobiology. Neuron 2007; 56: 422-37.

4. Jellinger KA. Rett Syndrome -- an update. J Neural Transm 2003; 110: 681-701.

5. Pierson J, Mayhew JF. Anesthesia in a child with Rett syndrome: a case report and literature review. AANA J 2001; 69: 395-6.

6. Dearlove OR, Walker RW. Anaesthesia for Rett syndrome. Paediatr Anaesth 1996; 6: 155-8.

7. Downs J, Bergman A, Carter P, Anderson A, Palmer GM, Roye D, et al. Guidelines for management of scoliosis in Rett syndrome patients based on expert consensus and clinical evidence. Spine (Phila Pa 1976) 2009; 34: E607-17.

8. Tofil NM, Buckmaster MA, Winkler MK, Callans BH, Islam MP, Percy AK. Deep sedation with propofol in patients with Rett syndrome. J Child Neurol 2006; 21: 210-3.

9. Konarzewski WH, Misso S. Rett syndrome and delayed recovery from anaesthesia. Anaesthesia 1994; 49: 357.

10. Seavello J, Hammer GB. Tracheal intubation in a child with trismus pseudocamptodactyly (Hecht) syndrome. J Clin Anesth 1999; 11: 254-6.

11. Guyuron B, Dinner MI. Bronchoscopic intubation of patients with trismus. Ann Plast Surg 1983; 10: 386-90. 